\title{
Achieving Large-Scale Quality Improvement in Primary Care Annual Wellness Visits and Hierarchical Condition Coding
}

\author{
Todd M. Zeiger, $M D^{7}$, Esther J. Thatcher, $P h D, R N^{7}$, Sona Kirpekar, $M D^{7}$, \\ Justin J. Coran, $\mathrm{PhD}, \mathrm{MPH}^{1,2}$, George Topalsky, $\mathrm{MD}^{7}$, Mary Jane D. Zarach, MBA', \\ Deanna A. Cox, MSN, MBA, RN ${ }^{7}$, Mark E. Schario, MS, RN, FACHE' , Kelsey A. Fuller, BA', \\ Patricia M. Upton, MPH, RT(R), CPhT ${ }^{7}$, Tomasina M. Green, MPH ${ }^{7}$, and Peter J. Pronovost, \\ $M D, \operatorname{PhD}^{1,2,3}$ (D)
}

'University Hospitals, Cleveland, OH, USA; ${ }^{2}$ School of Medicine, Case Western Reserve University, Cleveland, OH, USA; ${ }^{3}$ Frances Payne Bolton School of Nursing and Weatherhead School of Management, Case Western Reserve University, Cleveland, OH, USA.

\begin{abstract}
:
BACKGROUND: Completion of Medicare Annual Wellness Visits (AWV) and documentation of Hierarchical Condition Categories (HCC) are important metrics in accountable care organizations (ACO) with quality and financial implications. To improve performance in large healthcare organizations, quality improvement (QI) efforts need to be scaled up in a way that is feasible within available system-wide resources.

OBJECTIVE: We describe a 3-year effort using a multifaceted QI framework called the fractal management system for AWV and HCC performance.

DESIGN: Pre-post evaluation of a multi-level, health system-wide QI management system intervention between 2018 and 2020. The system provided project management, coaching, communications, feedback of performance, and health informatics.

PARTICIPANTS: The intervention was delivered to all 97 primary care practices within an Ohio-based accountable care organization, comprising 72,603 attributed Medicare and Medicare Advantage patients as of 2018. Eighty-nine of these practices were included in the analysis.

APPROACH: AWV completion was defined as percent of eligible patients with a documented AWV during the calendar year. HCC completion was defined as documented reassessment of all prior-year HCC conditions.

KEY RESULTS: AWV completion at the practice level increased from $23.7 \%$ (SD . 14) in 2018 to $34.9 \%$ (SD . 18) in 2019 , and $59.8 \%$ (SD .17) in 2020. This was a statistically significant effect of time on AWV completion rates overall $(F[2,87]=164.43, p<.000)$. More than half $(56.2 \%)$ of practices met or exceeded the $60 \%$ goal in 2020 . Practicelevel HCC completion tracking started in 2019 (M = $75.9 \%$, SD 7.4\%) and increased in 2020 ( $\mathrm{M}=79.7 \%$, SD 7.1\%); $t(172)=2.0, p<.001$.

CONCLUSIONS: AWV and HCC performance goals were met in 2020, despite service disruptions due to COVID19. The GI approach we used is applicable to other problems and other large healthcare systems.
\end{abstract}

Received March 2, 2021

Accepted December 13, 2021

Published online January 19, 2022
KEY WORDS: Annual Wellness Visit; Hierarchical Condition Category; implementation; Medicare; primary care.

J Gen Intern Med 37(6):1457-62

DOI: $10.1007 / \mathrm{s} 11606-021-07323-1$

(C) The Author(s) under exclusive licence to Society of General Internal Medicine 2022

\section{INTRODUCTION}

The Annual Wellness Visit (AWV) is a Medicare program that promotes patient visits that focus on preventive services such as vaccinations, bone density tests, and screening tests for cancer, chronic conditions, and cognitive problems. ${ }^{1}$ While long-term benefit of the AWV to patients' health outcomes lacks evidence, ${ }^{2,3}$ there is evidence of other benefits that may lead to improved outcomes. In an analysis of national Medicare claims for 2011-2014, patients who had an AWV received $62 \%$ more preventive services than patients without an AWV. ${ }^{4}$ Another national sample of Medicare patients who had an AWV showed lower Medicare expenditures than those without an AWV, though the savings mechanism was unclear. ${ }^{5}$ Though the proportion of eligible Medicare beneficiaries receiving an AWV increased from 8 to $23 \%$ between 2011 and 2016, acceleration is needed to increase Medicare patients who receive this beneficial covered service. ${ }^{5}$

Accurate and detailed documentation of patient conditions is increasingly important for managing care at a population level, and for adjusting expectations for the cost of care. Hierarchical Condition Categories (HCC) are used by the Centers for Medicare and Medicaid to calculate a risk adjustment factor for patients based on their diagnoses and demographics. ${ }^{6}$ Each HCC condition must be documented yearly. ${ }^{7}$ The AWV is ideal for providers to complete HCC documentation. HCC documentation is an important quality metric and also has substantial financial implications when caring for sicker patients. ${ }^{7}$

Improvement to AWV visit completion and coding of $\mathrm{HCC}$ for eligible patients increases provider quality metrics as well 
as revenue for Medicare Advantage and Medicare Shared Savings programs. However, published studies describing how to achieve improvements are sparse. We identified only two published papers that described efforts to improve rates of $\mathrm{AWV}^{8,9}$ and no studies related to HCC improvement. These small studies lacked an implementation framework, making it difficult to replicate. In this paper, we describe our 3-year efforts using a multifaceted QI framework to improve AWV and $\mathrm{HCC}$ rates in a large cohort of patients in a Medicare Advantage ACO receiving primary care services within an academic health system.

\section{METHODS}

\section{Setting}

University Hospitals of Cleveland (UH) ACO served around 554,525 active patients as of 2020. UH ACO patients are attributed across 16 value-based contracts with insurers, including traditional Medicare, Medicare Advantage, Medicaid, and a range of commercial providers. The UH ACO contracts identify and reward achievement of quality and utilization targets, particularly for care delivered by our primary care providers.

UH organizes primary care services through its Primary Care Institute (PCI). The PCI comprises a total of 472 providers (family physicians, internal medicine physicians, and advanced practice providers) in 97 practice groups located across a $>10,000-\mathrm{km}^{2}$ area in northeast Ohio. In 2018, all of these practices were paid on a relative value unit (RVU) model. Most practices had limited support staff.

\section{Intervention}

We used a previously published quality improvement (QI) framework for managing large complex projects. ${ }^{10-12}$ This fractal management framework (Fig. 1) provided a foundation for a QI management system to communicate priorities and progress, create conditions for success, and actively seek stakeholder engagement. The intervention to improve our AWV and HCC performance (Fig. 2) was designed with strategies across four domains of the fractal management system:

1. Declare goals

2. Create an enabling infrastructure

3. Engage clinicians and connect in peer learning activities

4. Report transparently and ensure shared accountability

Declare Goals. The AWV visit completion goal was set at 60\% among eligible Medicare and Medicare Advantage patients by the end of 2020 . We chose the $60 \%$ goal based on published literature, discussions with payers regarding excellent performance, and feasibility given our 2018 performance, believing that with application of the fractal management system, we could achieve such increase. The goal for HCC completion was set at 80\% in 2020. In 2019 and 2020, the UH ACO and PCI created financial incentives for achieving ACO goals, including for AWVs and HCC. Incentives included a $\$ 1000$ bonus to each physician and $\$ 1000$ per physician to distribute as a bonus for linked nonphysician staff when the physician and staff unit achieved AWVs for at least $60 \%$ of their assigned patients.

These goals were declared and communicated through multiple methods including meetings and emails. We required every provider to sign an attestation of receipt and understanding of the goals and incentives.

Create Enabling Infrastructure. Completing AWVs and HCC was primarily a work burden of individual practices and providers. To reduce this burden and enable the attainment of these QI goals, we created and deployed multiple levels of infrastructure and resources, including project management, coaching, communications, feedback of performance, and health informatics.

The ACO provided project management and direct support to practices primarily through two staff teams: Practice Transformation Facilitators (PTFs), typically healthcare specialists with master's degrees, and Practice Navigators, typically medical assistants. Practice Navigators systematically identified patients lacking an AWV and contacted them to schedule care. Each PTF worked with 20-30 practices, helping the staff realize goals by assessing, educating, and implementing best practices tailored for each practice. Practices were encouraged to try local tests of change to identify what works best for them. The PTF team provided a playbook of tools such as workflows for AWV and HCC completion, one pagers and tips sheets, and links to internal educational resources. These documents detailed identifying patients needing a wellness visit such as pre-visit scheduling tactics, how to schedule traditional Medicare versus Medicare Advantage due to different annual versus calendar year requirements, phone call scripting, point of service daily schedule review for opportunities, using electronic medical record wellness templates, ordering of screening and prevention services, and billing of these visits. In late 2020, this playbook evolved into a comprehensive repository or "e-book" of tools and resources that was electronically disseminated to primary care providers, available on UH's intranet, and updated regularly.

Two key feedback systems were monthly scorecards and data dashboards. The QI team provided monthly individualized scorecard reports to providers and practices that summarized performance year to date and forecasted targets in order to realize goals by the year end. Practices also received monthly reports with practice-level AWV performance, a list of attributed patients who still needed their yearly AWV, and time-sensitive AWV or HCC announcements. These reports were reinforced through a weekly "Tuesday Tips" concise email newsletter sent to all providers, office staff, and support staff. The newsletter highlighted top-performing practices and 


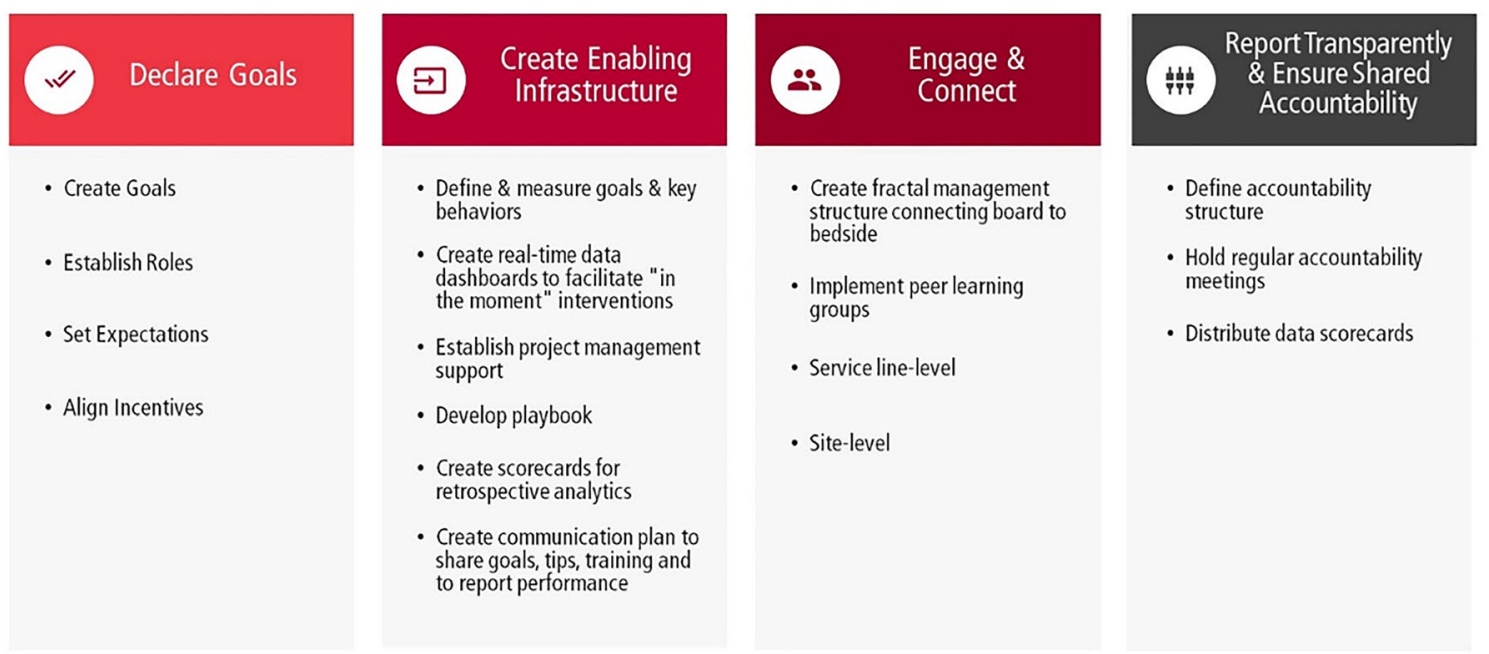

Figure 1 Fractal management system for quality improvement.

providers as a positive social motivator. A custom-built Power Business Intelligence dashboard allowed all team members to monitor real-time data and create actionable, customized patient reports filtered by practice, provider, and support roles.

As an additional health informatics tool to help make HCC gaps visible in the EMR, UH deployed a commercial technology (Clinovations). The Clinovations tool was able to identify gaps based on prior-year problem list/conditions billed compared to current year to date claims and provide "suspect" conditions based on lab data from the EMR and outside claims data of conditions not currently assessed in the EMR.

Engage and Connect Teams in Peer Learning. Engagement of the full organizational matrix structure was essential for bidirectional sharing of information, peer learning, and accountability among the large number of dispersed providers (see appendix). ${ }^{10,12,13}$ The PCI was led clinically by a primary care physician at a vice president level with regional medical directors. The PCI quality medical director was the primary person responsible for achieving the AWV and $\mathrm{HCC}$ performance goals. Each practice group had a medical site lead, and regional medical directors served as conduits of information for peer learning between the practice leads and PCI leadership. Parallel administration directors and practice managers provided additional opportunities for peer learning. A QI leadership team met weekly and comprised the PCI quality medical directors and staff representing the $\mathrm{ACO}$ and PCI.

A clinically active primary care physician leader was given dedicated non-clinical time to serve as a peer educator for providers and practice teams either referred by the PTF team or self-referred in response to monthly performance reports. Peer educational sessions lasted $30-45 \mathrm{~min}$ and focused on using data reports and creating efficient team workflows. Follow-up sessions could be scheduled if needed.

\section{Report Transparently and Ensure Shared Accountability.} Transparent reporting of reliable data, from a Microsoft Power Business Intelligence database, was disseminated through mass communications, individual messages through
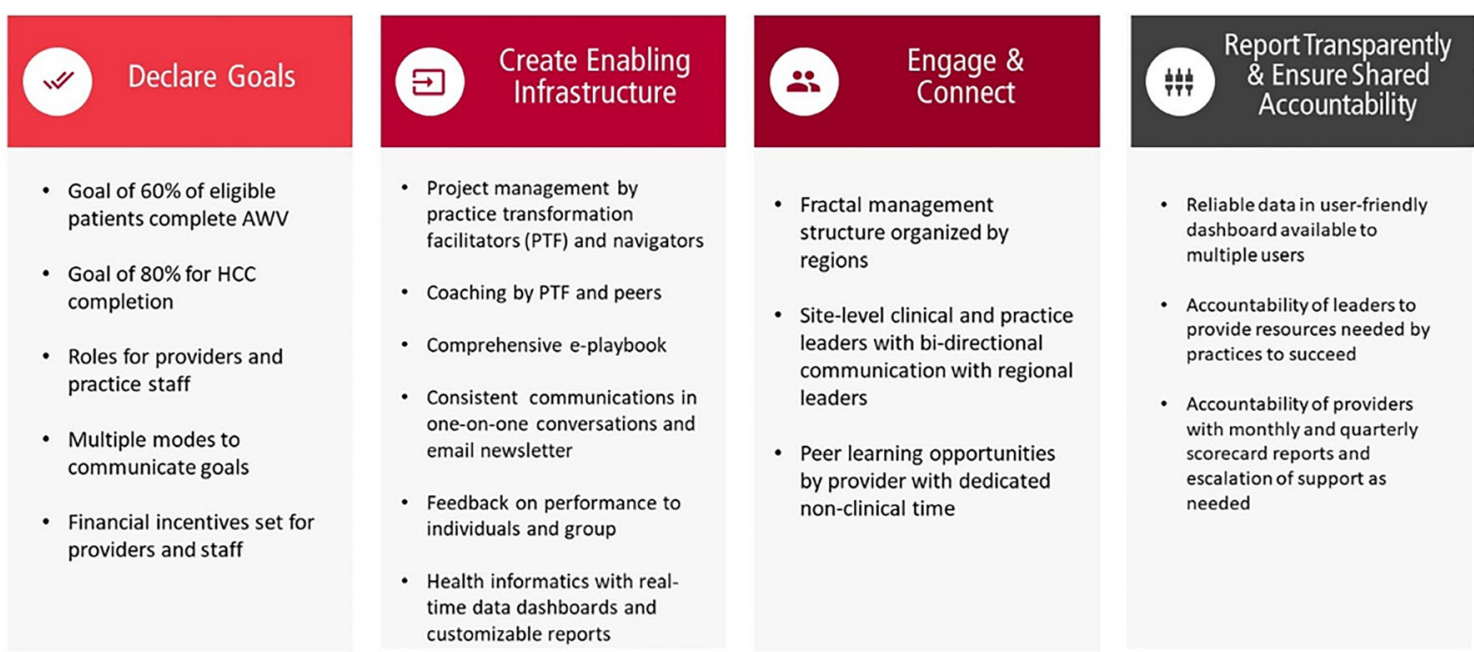

- Fractal management structure organized by regions

- Site-level clinical and practice leaders with bi-directional communication with regional leaders

- Peer learning opportunities by provider with dedicated non-clinical time
Reliable data in user-friendly dashboard available to multiple users
- Accountability of leaders to provide resources needed by practices to succeed
- Accountability of providers with monthly and quarterly scorecard reports and escalation of support as needed

Figure 2 Application of the fractal management system for quality improvement in AWV and HCC projects. 
the organizational leadership structure, and internal reports generated by practice staff. In addition, we created mechanisms to improve shared accountability. Shared accountability began with interventions to help ensure practices' success in achieving goals. Next, the PCI president and director of quality met quarterly with physicians who were far behind the expected performance. Practices who were on target after quarter two received a congratulation letter from health system leaders. Practices not meeting targets received a letter reiterating goals, offering support to help improve, and recognizing that many of their peers were on target.

\section{Study Design and Cohort}

Practices were studied longitudinally; patient samples were measured in a multiple time series cross-sectional design with the year as the unit of time. All $97 \mathrm{UH}$ primary care practice groups received the QI intervention. The analysis for this study included practices that in 2018 served at least 50 attributed Medicare and Medicare Advantage patients. Eighty-nine of 97 practice groups met these inclusion criteria. These practices and the number of providers $(n=324)$ remained stable in 2018-2020. Practice types were divided between family medicine and internal medicine specialties; no practices were primarily geriatric-focused. Patient inclusion criteria were adults 18 years of age and older with 2020 ACO membership dates, and no 2020 date of death in medical records.

This QI effort and evaluation was developed and carried out as part of regular health system operations. The goals of this study were part of ACO and PCI management goals. Use of the AWV and HCC were standard practice for all primary care patients pertaining to eligible populations. The UH Institutional Review Board determined this study was not considered human research.

\section{Outcome Measures}

The performance metrics evaluated were AWV completion and HCC coding or "gap closure." We defined AWV performance as the percent of our study population with a documented AWV in the EMR during the calendar year. The AWV performance metric combined Initial Preventive Physical Examination (IPPE or "Welcome to Medicare" visit), Initial Annual Wellness Visit, and Subsequent Annual Wellness Visit. Only one of these visits can occur for a patient each year, and despite some differences in requirements, they achieve the same purpose for health risk assessment, prevention services, and screening for conditions. We defined HCC completion as documented reassessment of all prior-year HCC conditions as either still active or resolved during the calendar year. We used Clinovations to define eligible HCC gaps.

\section{Data Sources and Analysis}

The primary data source was the Enterprise Data Warehouse at UH. This data warehouse assembled patient data from the electronic medical record and scheduling, and financial systems. We analyzed and reported AWV completion rates at patient and practice levels for 2018-2020. The HCC completion rate was analyzed through Clinovations; these data were available only for 2019 and 2020. We compared HCC rates using $t$ tests. We used repeated-measures ANOVA to test changes in AWV rates across three annual time points. We used IBM SPSS Statistics for Windows, Version 25.0, for all analyses.

\section{Impact of COVID-19}

In March 2020, due to COVID-19, 80\% of primary care visits were converted to telehealth encounters. This created significant challenges for Annual Wellness Visits due to the required elements of measuring blood pressure and weight (all AWVs) and visual acuity (IPPEs only). This required primary care practices to overcome a backlog of visits once in-person visits resumed in June 2020.

\section{RESULTS}

The overall AWV completion rate at the patient level was $24.9 \%(18,033$ of 72,476$)$ in $2018,35.2 \%(29,154$ of 82,781$)$ in 2019 , and $60.7 \%(52,872$ of 87,064$)$ in 2020 (Table 1). Similar results for AWV completion were found at the practice level: $23.7 \%$ (SD .14) in 2018, 34.9\% (SD .18) in 2019, and $59.8 \%$ (SD .17) in 2020. This was a statistically significant effect of time on AWV completion rates, $F(2,87)=164.43, p$ $<.000$. Post hoc analysis with a Bonferroni adjustment also showed significant increases from each year to the next: 20182019 (.112 [95\% CI .086-.138], $p<.000) ; 2019-2020$ (.249 [95\% CI .200-.298], $p<.000)$; and 2018-2020 (.361 [95\% CI $.310-.411], p<.000)$. In 2020,50 out of 89 practices $(56.2 \%)$ achieved the goal of $60 \%$ or greater AWV completion rate (Fig. 3). The overall HCC gap closure at the patient level was $75.9 \%(53,575$ of 70,606$)$ in 2019 and $80.2 \%(63,390$ of $79,036)$ in 2020 . This was a significant increase in practicelevel HCC scores from 2019 ( $\mathrm{M}=75.9 \%$, SD 7.4\%) to 2020 $(\mathrm{M}=79.7 \%, \mathrm{SD} 7.1 \%) ; t(172)=2.0, p<.001$. As a measure of

Table 1 AWV Performance for Practice-Level and Patient Population, 2018-2020

\begin{tabular}{llll}
\hline \hline & $\mathbf{2 0 1 8}$ & $\mathbf{2 0 1 9}$ & $\mathbf{2 0 2 0}$ \\
\hline Practices & 89 & 89 & 89 \\
Providers & 324 & 324 & 324 \\
Patients received AWV & 18,033 & 29,154 & 52,872 \\
Patients eligible for AWV & 72,476 & 82,781 & 87,064 \\
Mean AWV performance for patient & $24.9 \%$ & $35.2 \%$ & $60.7 \%$ \\
population & & & \\
Mean AWV performance by practice & $23.7 \%$ & $34.9 \%$ & $59.8 \%$ \\
\hline
\end{tabular}




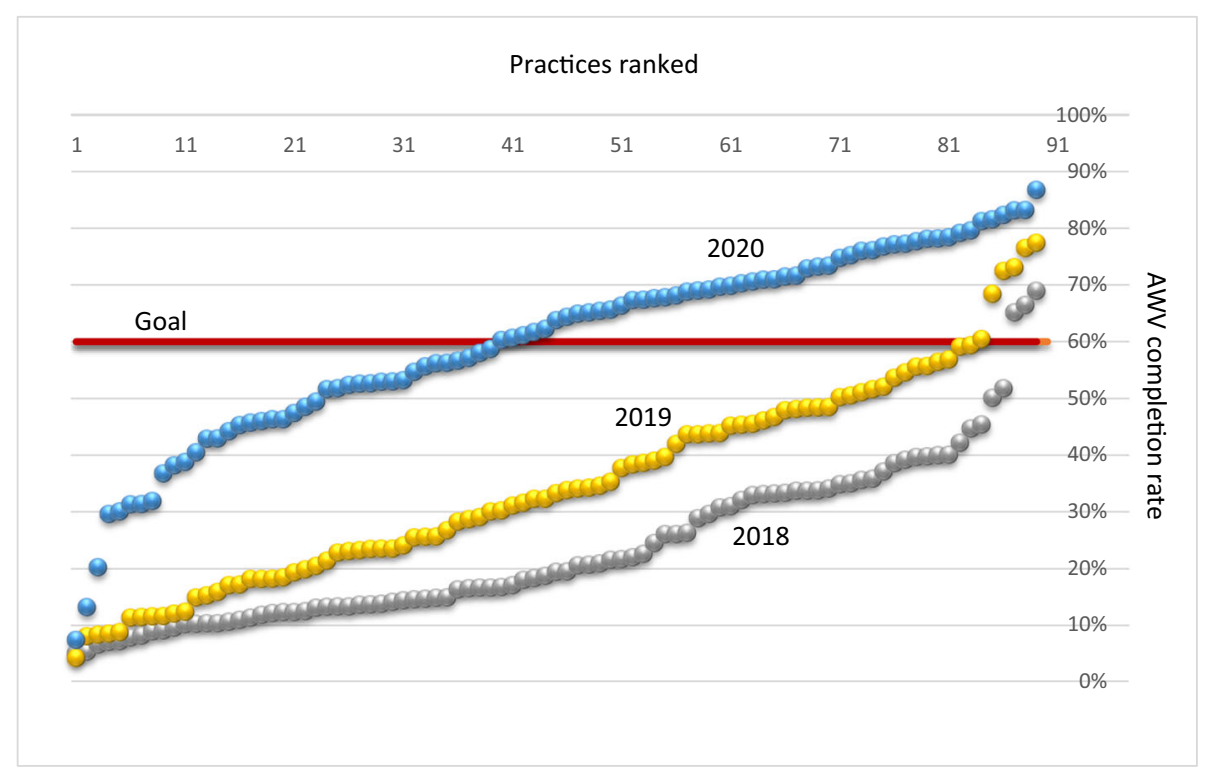

Figure 3 AWV performance 2018-2020 for rank ordered by practices $(n=89)$.

Footnote: The figure shows the distribution of practices for each study year ranging from the lowest to the highest AWV completion rate.

provider engagement, the weekly "Tuesday Tips" email had an open rate of over $70 \%$.

\section{DISCUSSION}

To achieve high-quality, equitable primary care delivery, large healthcare systems need effective approaches for engaging diverse, geographically dispersed primary care practices in improving their care. Universal access to AWVs is a cornerstone for ensuring equitable, comprehensive completion of needed prevention activities, including patient education, across enrolled patient populations. In this paper, we found that using a fractal management system for QI was associated with a significant improvement in practice-level performance on AWV visits from $23.7 \%$ in 2018 to $59.8 \%$ in 2020 . We also found increased closure of HCC gaps in primary care that likely was enabled by AWV completion, potentially enabling more comprehensive care.

Despite losing 3 months of time to schedule and deliver required in-person elements of $\mathrm{AWV}$ s due to the pandemic, primary care practices engaged in the QI intervention were still able to overcome the backlog of incomplete AWVs and meet these goals.

This study was funded and carried out entirely by the study healthcare system. As such, the fractal management system used represents a potentially feasible and acceptable approach for advancing QI across diverse primary care practices that do not themselves have the necessary resources for supporting major improvement initiatives. Our approach leaves room for innovation and tailoring directed at an individual practice's context, while providing needed materials, education, data resources, and practice leader release time from usual duties. This QI system pre-existed the AWV and HCC interventions and remains in place for addressing other system-wide issues.
This study fills an important gap in published evidence on methods for improving AWV and HCC performance. Of the few previous publications found, one study involved a series of plan-do-study-act(PDSA) cycles in a small non-ACO practice group that engaged nurse case managers to recruit patients and perform the AWVs. ${ }^{9}$ Another study described ACO members' approaches to deliver preventive services such as AWVs, but did not apply performance improvement interventions. ${ }^{8}$ The improvements in our study affected a larger population than other published studies.

A strength of our study was the use of an explicit QI framework, providing greater confidence in the replicability of the intervention and its relationship to the observed outcomes. This fractal management system seeks to balance local quality improvement efforts and innovation with system-wide efforts and accountability. ${ }^{14}$ Local QI models, such as PDSA or the Model for Improvement, ${ }^{15}$ are helpful for identifying, contextualizing, and revising effective intervention; however, they do not provide a framework for large-scale change across a system. Our fractal management system builds upon and supports local QI models by providing a framework for spreading QI interventions and ensuring shared accountability for performance across large and complex health systems. Our approach can help large systems organize and support improvements that both integrate priorities and experiences of individual practices and reflect the priorities and resources of the whole system. ${ }^{14}$

Another strength was communication among stakeholders. We intentionally addressed the "adaptive" side of change, ${ }^{16}$ by focusing on learning rather than judging, encouraging rather than shaming, and celebrating rather than punishing. We also made efforts to build trust by co-creating the interventions with the care teams and doing things "with rather than to" them. In all of our communications, we sought mutual respect 
and mindfully used words demonstrating power with rather than power over the care teams. Third, we had strong collaboration between the ACO team, PCI leadership, and physician practices. In addition, we used the transparent reporting and awareness of practices lagging in achievement of goals as an opportunity to have focused peer (physician) contact to inquire about concerns or barriers and offer direct education sessions to practice teams. Feedback of performance data has a long history of success in promoting improvement. ${ }^{17}$

Future directions focus on sustainability of the QI initiatives and utilizing lessons learned. Sustainability of this intervention depends significantly on user acceptability. Stakeholders' perceptions of the AWV and HCC QI projects were not directly measured. However, our health system continues to support the fractal management system described here across several clinical performance improvement targets in 2021, and as an approach to eliminating "defects in value"11, 18 across our ACO and the entire UH health system. Goals for 2021 are provider-based and have increased to $65 \%$ for $\mathrm{AWV}$ and $90 \%$ for HCC.

Our study has limitations. First, we used an observational, cross-sectional study design without a control group and as such cannot make causal inferences between the intervention and outcomes. However, we found no published literature suggesting broad improvements over time and our payers stated that they have not seen systematic improvements during the timeframe of this study. In addition, the financial incentives from payers to improve these metrics did not change during the study (other than as part of our intervention). Second, our patient population analysis lacked demographic details such as race/ethnicity, gender, socioeconomic status, and other factors from which to evaluate equitable access to AWVs. Third, we did not report AWV or HCC monthly control charts due to limitations with retrospective data extraction, though this level of detail would be valuable. Fourth, our study focused only on completion rates for AWVs and HCCs and did not evaluate patient health outcomes. Fifth, we studied only one health system in the Midwest and our results may not be generalizable to other systems.

\section{CONCLUSION}

The use of a multifaceted framework as the foundation for a management and accountability system was associated with significant improvements in AWV and HCC gap closure between 2018 and 2020 despite the COVID-19 pandemic. Other large health systems could apply this framework and strategies to improve performance in these or other quality metrics.

Acknowledgements: Funding for this project was provided internally by University Hospitals.
Corresponding Author: Peter J. Pronovost, $M D, P h D$; University Hospitals, Cleveland, OH, USA (e-mail: Peter.Pronovost@UHhospitals. org).

\section{Declarations:}

Conflict of Interest: The authors declare that they do not have a conflict of interest.

\section{REFERENCES}

1. Colburn JL, Nothelle S. The Medicare Annual Wellness Visit. Clin Geriatr Med. 2018;34(1):1-10. https://doi.org/10.1016/j.cger.2017.09.0011.

2. Beckman AL, Becerra AZ, Marcus A, DuBard CA, Lynch K, Maxson E, et al. Medicare Annual Wellness Visit association with healthcare quality and costs. Am J Manag Care. 2019;25(3):e76-e82.

3. Simpson VL, Kovich M. Outcomes of primary care-based Medicare annual wellness visits with older adults: A scoping review. Geriatr Nurs. 2019;40(6):590-6.

4. Camacho F, Yao NA, Anderson R. The effectiveness of Medicare Wellness Visits in accessing preventive screening. J Prim Care Community Health. 2017;8(4):247-255. https://doi.org/10.1177/2150131917736613

5. Misra A, Lloyd JT. Hospital utilization and expenditures among a nationally representative sample of Medicare fee-for-service beneficiaries 2 years after receipt of an Annual Wellness Visit. Prev Med. 2019;129: 105850. https://doi.org/10.1016/j.ypmed.2019.105850

6. Yeatts JP, Sangvai D. HCC coding, risk adjustment, and physician income: what you need to know. Fam Pract Manag. 2016;23(5):24-7.

7. Champlin G, Pitsikoulis J. Impact of coding and documentation on risk scores. In: Gross PA, ed. Pathways to a Successful Accountable Care Organization. Johns Hopkins University Press; 2020:203-212:chap 10.

8. Briggs ADM, Fraze TK, Glick AL, Beidler LB, Shortell SM, Fisher ES. How do Accountable Care Organizations deliver preventive care services? A mixed-methods study. J Gen Intern Med. 2019;34(11):2451-2459. https://doi.org/10.1007/s11606-019-05271-5

9. Bluestein D, Diduk-Smith R, Jordan L, Persaud K, Hughes T. Medicare Annual Wellness Visits: how to get patients and physicians on board. Fam Pract Manag. 2017;24(2):12-16.

10. Pronovost PJ, Armstrong CM, Demski R, et al. Creating a high-reliability health care system: improving performance on core processes of care at Johns Hopkins Medicine. Acad Med;90(2):165-72. https://doi.org/10. 1097/ACM.0000000000000610

11. Pronovost P, Urwin, J., et al. Making a dent in the trillion-dollar problem: toward zero defects. NEJM Catalyst Innovations in Care Delivery. 2021;2(1). https://doi.org/10.1056/CAT.19.1064

12. Pronovost PJ, Marsteller JA. Creating a fractal-based quality management infrastructure. J Health Org Manag. 2014. https://doi.org/10. 1108/JHOM-11-2013-0262.

13. Kravet SJ, Bailey J, Demski R, Pronovost P. Establishing an ambulatory medicine quality and safety oversight structure: leveraging the fractal model. Acad Med. 2016;91(7):962-6. https://doi.org/10.1097/ACM. 0000000000001102

14. Caron A, Pronovost P. Central versus local quality efforts: the need for both. J Am American Board Fam Med. 2021;34(5):1038-41. https://doi. org/10.3122/jabfm.2021.05.210015.

15. Langley G, Moen R, Nolan K, Nolan T, Norman C, Provost L. The improvement guide: a practical approach to enhancing organizational performance. 2nd ed. Jossey-Bass; 2009.

16. Heifetz RA, Laurie DL. The work of leadership. Harv Bus Rev. 1997;75(1):124-34

17. Tierney WM. Improving clinical decisions and outcomes with information: a review. Int J Med Inform. 2001;62(1):1-9. https://doi.org/10.1016/ S1386-5056(01)00127-7.

18. Runnels P, Wobbe HM, Pronovost PJ. Eliminating defects in behavioral health treatment. Psychiatr Serv. 2021;72(2):213-215. https://doi.org/ 10.1176/appi.ps.202000255

Publisher's Note Springer Nature remains neutral with regard to jurisdictional claims in published maps and institutional affiliations. 